Supporting information for the manuscript:

\title{
A thiamine-dependent enzyme utilizes an active tetrahedral intermediate in vitamin $\mathrm{K}$ biosynthesis
}

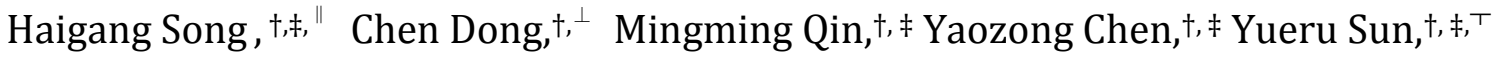

Jingjing Liu, $§$ Wan Chan,, Z Zhihong Guo*十, ‡

†Department of Chemistry, ¥State Key Laboratory for Molecular Neuroscience,

§Environmental Science Program, The Hong Kong University of Science and Technology, Clear Water Bay, Kowloon, Hong Kong SAR, China.

\section{This file includes:}

Materials and Methods

Tables S1-S2

Figures S1-S6

Supplementary references 


\section{Materials and Methods}

Materials. Thiamine diphosphate (ThDP), $\mathrm{NAD}^{+}$, pyruvate, $\alpha$-ketoglutaric acid, magnesium chloride, manganese (II) sulfate, 1-thio- $\beta$-D-galactopyranoside, salts, PEG 3350, PEG MME 5000, glycerol and buffers were purchased from Sigma-Aldrich. Restriction enzymes and T4 DNA ligase were purchased from New England Biolabs. UV-vis absorbance was measured using a UV-1800 spectrophotometer from Shimadzu Corporation. PCR amplification and sitedirected mutagenesis were conducted with a PTC-200 Peltier thermal cycler from MJ Research. Protein chromatography was done using an ÄKTA Protein Purification System from GE Healthcare Life Sciences. HPLC analysis and purification were carried out using a Waters $600 \mathrm{E}$ system with model 2487 dual $\lambda$ absorbance detector. ${ }^{1} \mathrm{H}-\mathrm{NMR}$ experiments were performed using a Bruker AVIII 400 NMR spectrometer. Primers were ordered from Life Technologies and DNA sequencing was done by BGI. PyMol was used to generate all the structure graphics. ${ }^{1}$

Expression and purification of MenD. The gene of E. coli menD was amplified from $\mathrm{pETM} \mathrm{MenD}^{2}$ and subcloned into pET28a(+) between $\mathrm{NcoI}$ and XhoI restriction site for expression as an untagged protein with two extra residues (Met-Ser) at the N-terminus. The primers used in this gene amplification were CCCGCCATGGCAATGTCAGTAAGCGCAT TTAA (menD forward) and CCGCCTCGAGTCATAAATGGCTTACACTCG (menD reverse). The recombinant plasmid was introduced into BL21 (DE3) for growth in LB medium supplemented with $50 \mu \mathrm{g} / \mathrm{ml}$ kanamycin at $37{ }^{\circ} \mathrm{C}$. The protein was expressed at $18{ }^{\circ} \mathrm{C}$ for $18 \mathrm{~h}$ by addition of $0.1 \mathrm{mM}$ IPTG when $\mathrm{OD}_{600}$ reached 0.8 . The wild-type MenD protein was first precipitated from the crude extract with $40 \%$ saturated ammonium sulfate (about $1.57 \mathrm{M}$ ) and then re-dissolved in $12 \mathrm{ml}$ of $25 \mathrm{mM}$ Tris- $\mathrm{HCl}$ ( $\mathrm{pH}$ 8.0) buffer containing $10 \%$ glycerol. After salt removal with a HiPrep 26/10 Desalting column (GE Healthcare), the protein solution was loaded on to a HiPrep DEAE FF 16/10 column (GE Healthcare) for purification with a linear gradient of $100 \%$ buffer A ( $25 \mathrm{mM}$ Tris- $\mathrm{HCl}(\mathrm{pH} 8.0)$ solution containing $10 \%$ glycerol) to $100 \%$ buffer B (25 mM Tris- $\mathrm{HCl}$ (pH 8.0) solution containing $1 \mathrm{M} \mathrm{NaCl}$ and $10 \%$ glycerol). The MenD-containing fractions were collected at 10-12\% of buffer B, concentrated and loaded to a HiPrep Sephacryl S-200 column for further purification to near homogeneity with a purity > 95\% as estimated from an SDS-PAGE gel. The purified MenD solution was concentrated to $58 \mathrm{mg} / \mathrm{ml}$ and stored in $25 \mathrm{mM}$ Tris- $\mathrm{HCl}$ ( $\mathrm{pH} 8.0$ ) buffer containing $10 \%$ glycerol at $-20{ }^{\circ} \mathrm{C}$. In a typical purification, $240 \mathrm{mg}$ MenD was obtained from $3 \mathrm{~L}$ shake-flask culture.

Activity tests. The isochorismate substrate was prepared from EntC and chorismic acid, as described previously and purified using the reported methods with minor modification..$^{2-4}$ Typically, $4 \mathrm{mg}$ chorismic acid was incubated with $10 \mu \mathrm{M}$ EntC in $500 \mu \mathrm{l}$ of $50 \mathrm{mM}$ Tris- $\mathrm{HCl}$ ( $\mathrm{pH}$ 8.0) buffer solution containing $50 \mathrm{mM} \mathrm{NaCl}$ and $2 \mathrm{mM} \mathrm{MgCl}_{2}$ for $2 \mathrm{~h}$ at $37{ }^{\circ} \mathrm{C}$. Isochorismate was then loaded to an Xterra semipreparative C-18 reversed-phase column (10 
$\mu \mathrm{m}, 7.8 \times 300 \mathrm{~mm}$ ) and eluted with a linear gradient from $100 \%$ of buffer A (water containing $1 \%$ formic acid) to $100 \%$ of buffer B (acetonitrile containing $1 \%$ formic acid) over $45 \mathrm{~min}$ at a flow rate of $2.0 \mathrm{ml} / \mathrm{min}$ after 10 min washing with $100 \%$ buffer A on the Waters 600 HPLC system. Isochorismate-containing fractions with a retention time of 25.4 min were collected, lyophilized and frozen at $-80{ }^{\circ} \mathrm{C}$. In enzymatic assays, isochorismic acid was freshly dissolved in pure water and its concentration was determined by absorbance at $278 \mathrm{~nm}\left(\varepsilon=8300 \mathrm{M}^{-1} \cdot \mathrm{cm}^{-}\right.$ $\left.{ }^{1}\right) .{ }^{2}$ After purification, MenD was assayed to have a specific activity consistent with the reported value using a spectroscopic method monitoring isochorismate consumption at 278 $\mathrm{nm} .{ }^{2} \mathrm{Mg}^{2+}$ was found to be completely replaceable with $\mathrm{Mn}^{2+}$ at the same concentration in this assay without reducing the MenD activity. In other MenD activity tests, the SEPHCHC product was further converted to SHCHC for UV-vis detection at $290 \mathrm{~nm}$ using MenH as the coupling enzyme. 5,6

In a typical single turnover experiment, the post-decarboxylation intermediate was formed by adding $25 \mu \mathrm{M} \alpha$-ketoglutaric acid to a solution containing $50 \mu \mathrm{M} \mathrm{MenD}, 5 \mu \mathrm{M} \mathrm{MenH}, 100$ $\mu \mathrm{M}$ ThDP and $2 \mathrm{mM} \mathrm{MgSO}_{4}$ in $400 \mu \mathrm{l}$ of $100 \mathrm{mM}$ potassium phosphate buffer (pH 7.0). Under these conditions, the intermediate was formed almost instantly as monitored by circular dichroism spectroscopy. After mixing, the second substrate isochorismate was added to a final concentration of $60 \mu \mathrm{M}$ at different reaction time points and the reaction was subsequently quenched after $10 \mathrm{~min}$ by adding $100 \mu \mathrm{l}$ of $2 \mathrm{M} \mathrm{CF}_{3} \mathrm{COOH}$. MenH at the given concentration was tested to be capable of converting all SEPHCHC to SHCHC within a short period of time $(<1 \mathrm{~min})$ at room temperature. After removal of the precipitated protein by centrifugation, 390 $\mu \mathrm{l}$ of the reaction mixture was mixed with $10 \mu \mathrm{l}$ of $5.3 \mathrm{mM} \mathrm{NAD}^{+}$(internal standard) and the SHCHC product was then subjected to HPLC quantification after isocratic elution with an aqueous solution containing $1 \%$ formic acid on an analytical C-18 reversed-phase column. The control reaction was performed by simultaneous addition of the ketoacid and isochorismate substrates to the enzyme solution and quenched after $10 \mathrm{~min}$ incubation at room temperature.

SHCHC was chemoenzymatically synthesized as a standard in the HPLC analysis using a described method. ${ }^{5,6}$ In a typical preparation, $4 \mathrm{mg}$ chorismic acid was incubated with $10 \mu \mathrm{M}$ EntC, $25 \mu \mathrm{M}$ MenD and $10 \mu \mathrm{M}$ MenH in $500 \mu \mathrm{l}$ of $200 \mathrm{mM}$ potassium phosphate buffer $(\mathrm{pH}$ 7.4) containing $50 \mathrm{mM} \alpha$-ketoglutaric acid, $80 \mu \mathrm{M}$ ThDP and $7.5 \mathrm{mM} \mathrm{MgSO}_{4}$ at $37{ }^{\circ} \mathrm{C}$ for $3 \mathrm{~h}$. After removal of enzymes by ultrafiltration, SHCHC was eluted isocratically with an aqueous solution containing $1 \%$ formic acid at a flow rate of $2 \mathrm{ml} / \mathrm{min}$ on the Waters 600 HPLC system using an Xterra semipreparative $\mathrm{C}$-18 reversed-phase column $(10 \mu \mathrm{m}, 7.8 \times 300 \mathrm{~mm})$. The peak fractions containing SHCHC were collected, lyophilized and frozen at $-80{ }^{\circ} \mathrm{C}$.

Circular dichroism (CD) analysis of the intermediate formation. Static spectra were recorded on a JASCO J-815-150S CD spectrometer in a $10 \mathrm{~mm}$ path length cell in the nearUV (270-370 nm) wavelength region at a bandwidth of $2 \mathrm{~nm}$. In a typical experiment, $\alpha-$ ketoglutaric acid (final concentration: $2 \mathrm{mM}$ ) was added to a $100 \mathrm{mM}$ potassium phosphate 
buffer solution ( $\mathrm{pH} 7.0$ ) containing $2.2 \mathrm{mg} / \mathrm{ml}(33 \mu \mathrm{M}) \mathrm{MenD}, 200 \mu \mathrm{M}$ ThDP and $2 \mathrm{mM}$ $\mathrm{MgSO}_{4}$ and the mixture was immediately analyzed by the CD spectroscopy. The CD spectra were taken every $5 \mathrm{~min}$ and were not found to significantly change over a period of $30 \mathrm{~min}$ at room temperature. Control CD spectra were taken in the absence of $\alpha$-ketoglutaric acid with or without ThDP. The ThDP-bound intermediate gave rise to a species with a positive signal at $301 \mathrm{~nm}$ and a broad intense negative signal from 307-365 nm with double minima at $320 \mathrm{~nm}$ and $330 \mathrm{~nm}$, which are absent in the enzyme with or without the ThDP cofactor. These observed spectral features are clearly different from those of the enamine intermediate in transketolase, which shows positive circular dichroism signals in the ranges of 290-295 nm and $>300 \mathrm{~nm}$ (Fiedler et al. Proc. Natl. Acad. Sci. USA 2002, 99, 591.).

To determine what is formed in the reaction of $\alpha$-ketoglutaric acid with MenD, the reaction (with the scale as in the CD experiment) was carried out and quenched immediately with trifluoroacetic acid 20 seconds after addition of the keto-acid substrate at room temperature. After removal of the precipitated protein, the reaction mixture was analyzed by ESI-MS with a spectrum as shown in Fig. S5(A). Only the succinic semialdehyde-ThDP adduct (see below) and free ThDP were detected in the mixture, while the predecarboxylation ThDP adduct (molecular formula: $\mathrm{C}_{17} \mathrm{H}_{25} \mathrm{~N}_{4} \mathrm{O}_{12} \mathrm{P}_{2} \mathrm{~S}^{+}$; calculated molecular weight: 571.07) was never detected.

Deuterium exchange of the $\mathbf{C}_{2} \boldsymbol{\alpha}$-associated proton of the intermediate. To examine the exchangeability of the $\mathrm{C}_{2 \alpha}$-associated proton, the postdecarboxylation intermediate was first prepared by incubating $500 \mu \mathrm{M}$ MenD with $500 \mu \mathrm{M} \alpha$-ketoglutaric acid, $500 \mu \mathrm{M}$ ThDP and 5 $\mathrm{mM} \mathrm{MgSO}_{4}$ in $2 \mathrm{ml}$ of $100 \mathrm{mM}$ phosphate buffer ( $\mathrm{pH} \mathrm{7.0)}$ solution. After 5 min reaction at room temperature, an equal volume of $\mathrm{D}_{2} \mathrm{O}$ was added to the reaction mixture and the resulting solution was further incubated for $30 \mathrm{~min}$ at room temperature to allow deuterium exchange before the reaction was quenched by addition of $1 \mathrm{ml}$ of $2 \mathrm{M} \mathrm{CF}_{3} \mathrm{COOH}$. After removal of the precipitated protein by centrifugation, the reaction mixture was concentrated by lyophilization and re-dissolved in a minimum amount of pure water. The succinic semialdehyde-ThDP adduct with a retention time of $6.2 \mathrm{~min}$ was then purified by HPLC from the reaction mixture using an Xterra semi-preparative C-18 reversed-phase column $(10 \mu \mathrm{m}, 7.8 \times 300 \mathrm{~mm})$ and isocratic elution with water containing $1 \%$ formic acid at a flow rate of $2 \mathrm{ml} / \mathrm{min}$. A small volume of the eluted fraction was taken out for ESI-MS analysis and the remaining portion was lyophilized and further re-dissolved in $200 \mu \mathrm{D}_{2} \mathrm{O}$ for ${ }^{1} \mathrm{H}-\mathrm{NMR}$ experiment. As a control, the reaction was carried out using an equal volume of $\mathrm{H}_{2} \mathrm{O}$ instead of $\mathrm{D}_{2} \mathrm{O}$ in the exchange experiment and the non-deuterated ThDP adduct was purified and analyzed in parallel. It was found that a significant amount of glycerol ended up in the purified ThDP adduct to interfere with the ${ }^{1} \mathrm{H}$ NMR analysis when MenD was purified and stored in buffers containing 10\% glycerol. To reduce this interference, MenD in the deuterium exchange experiments was purified freshly in glycerol-free buffers and used directly in the reactions. MenD freshly purified in the absence 
of glycerol was found to have the same activity as the enzyme stored in $10 \%$ glycerol in enzymatic assays.

In the mass spectrum of the isolated succinyl semialdehyde-ThDP, two molecular ions were detected at $\mathrm{m} / \mathrm{z}=527(\mathrm{M})$ and $528(\mathrm{M}+1)$. Assuming that the intensity ratio of the $(\mathrm{M}+1)$ : $\mathrm{M}$ is $R_{H}$ and $R_{D}$ before and after the exchange, respectively, the rate of deuterium incorporation is equal to $\left(R_{D}-R_{H}\right) /\left(1-R_{H}\right)$. This deuterium incorporation rate was also calculated from the decrease in the normalized intensity of $\mathrm{C} 2 \alpha-\mathrm{H}$ relative to $\mathrm{C} 6$ '- $\mathrm{H}$ in ${ }^{1} \mathrm{H}-\mathrm{NMR}$ spectrum of the isolated product from the deuterium exchange experiment.

Crystallization of MenD and soaking with the substrates. Initial screening of the crystallization conditions for the wild-type MenD was performed in the presence of ThDP and $\mathrm{Mn}^{2+}$ with the hanging-drop diffusion method at $289 \mathrm{~K}$ using commercially available screening kits, including Index Screen, PEG/Ion screen, Crystal Screen I and II from Hampton Research and Wizard Screen I and II from Rigaku Corporation. Subsequent optimization of the preliminary conditions led to plate-like crystals within 4 days with desirable diffraction qualities by mixing $1 \mu \mathrm{l}$ protein solution $(5 \mathrm{mg} / \mathrm{ml}$ protein supplemented with $0.5 \mathrm{mM}$ ThDP and $\left.5 \mathrm{mM} \mathrm{MnSO}_{4}\right)$ with $1 \mu \mathrm{l}$ reservoir condition $(0.16 \mathrm{M}$ magnesium formate, $1 \%$ Tascimate pH 7.0, 0.02 M HEPES pH 7.0, 14\% PEG 3350 and 2\% PEG MME 5000). These crystals were used in the soaking experiments (see below) to obtain most crystal structures shown in Table S1 (all but PDB ID: 5EJ8). Under the same conditions, crystals were found to continue to grow and form bigger single crystals with better diffraction quality and resolution after three months at $16{ }^{\circ} \mathrm{C}$. These later crystals were used to obtain the structure at $1.34 \AA$ resolution (PDB ID: 5EJ8). In these experiments, addition of $5 \mathrm{mM} \mathrm{MnSO}_{4}$ was found to be crucial to the growth and diffraction quality of the resulting crystals. As a result, $\mathrm{Mn}^{2+}$ was modeled as the metal ligand of the pyrophosphate moiety of the ThDP cofactor in the resulting structures despite the fact that the crystals were grown in the presence of magnesium ion at a much higher concentration.

In the soaking experiments, the grown single crystals were soaked in the cryo-protecting solution containing the ketoacid substrate (the mother liquor supplemented with $20 \%$ glycerol and $10 \mathrm{mM} \alpha$-ketoglutarate) for a time period ranging from $21 \mathrm{~s}$ to $1.5 \mathrm{~h}$. At different time points, the soaking reactions were stopped by directly transferring the crystals into liquid nitrogen for data collection.

To test the activity of the post-decarboxylation intermediate, the MenD single crystals were first soaked in $10 \mathrm{mM} \alpha$-ketoglutarate for $2 \mathrm{~min}$ to form the postdecarboxylation intermediate as described above. Subsequently, the resulting crystals were transferred into the mother liquor supplemented with $20 \%$ glycerol to remove the extra $\alpha$-ketoglutarate and then transferred into another cryo-protecting solution comprising of the mother liquor supplemented with $20 \%$ glycerol and $250 \mu \mathrm{M}$ isochorismate for a time period up to $20 \mathrm{~min}$ without damaging the crystals. At different time points, the crystals soaked with isochorismate were transferred to 
liquid nitrogen and frozen for data collection. The SEPHCHC product in the mother liquid was converted to SHCHC for UV-vis detection at $290 \mathrm{~nm}$ using MenH as the coupling enzyme. 5, 6 In the second soaking experiment, the MenD crystals cracked immediately when isochorismate concentration was $500 \mu \mathrm{M}$.

Data collection, structural determination and refinement. All the X-ray data were collected at BL17U beamline of the Shanghai Synchrotron Radiation Facility (SSRF) with an ADSC Quantum 315R charge-coupled device detector or BL19U of the National Center for Protein Science Shanghai (NCPSS) with a PILATUS3 6M detector at $100 \mathrm{~K}$. For the 1.34- $\AA$ resolution structure, the data were collected at different spots of the crystal to minimize radiation damage. Diffraction images were either processed with HKL2000 ${ }^{7}$ or indexed, integrated with iMosfilm ${ }^{8}$ and scaled with Aimless. ${ }^{9}$ All the structures were solved by molecular replacement with Phaser ${ }^{10}$ using E. coli MenD (PDB entry 2JLC) ${ }^{11}$ as initial search model. The generated model was further manually built by COOT $^{12}$ and refined by PHENIX. ${ }^{13}$ TLS and NCS were used in the refinement of all crystal structures. For the higher resolution structure at $1.34 \AA$ resolution (PDB ID: 5EJ8), a slightly different refinement procedure was followed with water molecules added at $1.5 \AA$ A resolution and re-checked at $1.34 \AA$ resolution. Hydrogen atoms of the residues were automatically generated using the riding model in PHENIX at $1.34 \AA$ A resolution. TLS and NCS refinement methods were used in the middle refinement cycles. Anisotropic atomic displacement parameter refinement for all the nonhydrogen atoms was applied in the final several refinement cycles. The restraints for the ligands were generated using PRODUG ${ }^{14}$ and optimized by eLBOW. ${ }^{15}$ The overall qualities of all the finalized structures were assessed by PROCHECK ${ }^{16}$ and MolProbity. ${ }^{17}$ The data statistics for collection and refinement of the structures are summarized in Table S1 with Ser391 as a Ramanchandran outlier as in other known MenD structures (PDB entries 2JLA, 2JLC, and 2X7J). 
Table S1. Data collection and refinement statistics

\begin{tabular}{|c|c|c|c|c|c|c|c|}
\hline & MenD-KG-2 min & MenD-KG-21 s & MenD-KG-15 min & MenD-KG-1.5 h & $\begin{array}{l}\text { MenD-KG-2 min- } \\
\text { Iso-2 min }\end{array}$ & $\begin{array}{l}\text { MenD-KG-2 min- } \\
\text { Iso-7min }\end{array}$ & $\begin{array}{l}\text { MenD-KG-2 min- } \\
\text { Iso-13 min }\end{array}$ \\
\hline PDB ID & $5 \mathrm{EJ} 8$ & 5EJ7 & 5EJ4 & 5EJ5 & $5 \mathrm{EJ} 6$ & 5EJA & 5EJ9 \\
\hline Space group & $P_{1}$ & $P_{1}$ & $P_{1}$ & $P_{1}$ & $P_{1}$ & $P_{1}$ & $P_{1}$ \\
\hline$a, b, c(\AA)$ & $90.66,90.76,169.34$ & $90.60,90.61,167.57$ & $90.79,90.77,169.56$ & $90.76,90.80,170.10$ & $90.48,90.51,171.64$ & $90.61,90.66,169.46$ & $90.72,90.72,169.47$ \\
\hline$\alpha, \beta, \gamma\left({ }^{0}\right)$ & $83.25,76.03,64.32$ & $76.08,83.45,63.44$ & $83.27,76.01,64.30$ & $75.79,83.29,64.34$ & $83.02,75.85,64.33$ & $76.03,83.39,64.41$ & $76.06,83.35,64.38$ \\
\hline Reflection & 2345165 (1002427) & $1250745(634680)$ & $874242(443335)$ & 371017 (194725) & $544093(213686)$ & $1146914(583261)$ & 962167 (487194) \\
\hline Redundancv & $2.3(2.4)$ & $2.0(2.0)$ & $2.0(2.0)$ & $1.9(1.9)$ & $2.5(2.4)$ & $2.0(2.0)$ & $2.0(2.0)$ \\
\hline Completeness (\%) & $94.5(92.4)$ & $96.0(91.3)$ & $96.6(94.8)$ & $92.4(95.5)$ & 93.7 (82.7) & $93.6(93.4)$ & $96.5(91.2)$ \\
\hline$I / \sigma(I)$ & $5.0(2.0)$ & $6.4(3.5)$ & $7.1(2.5)$ & $5.8(2.8)$ & $6.7(3.9)$ & $6.9(2.4)$ & $8.1(3.1)$ \\
\hline $\boldsymbol{R}_{\text {merge }}{ }^{a}$ & $0.123(0.409)$ & $0.063(0.192)$ & $0.077(0.417)$ & $0.101(0.240)$ & $0.120(0.213)$ & $0.070(0.303)$ & $0.048(0.156)$ \\
\hline \multicolumn{8}{|l|}{ Refinement } \\
\hline Resolution (A) & $27.65-1.34$ & $33.49-1.56$ & $41.82-1.77$ & $36.28-2.30$ & $34.89-2.24$ & $50.52-1.60$ & $41.85-1.72$ \\
\hline $\boldsymbol{R}_{\text {work }} / \boldsymbol{R}_{\text {free }}{ }^{b}$ & $0.1328 / 0.1636$ & $0.1458 / 0.1830$ & $0.1758 / 0.2169$ & $0.1830 / 0.2343$ & $0.1951 / 0.2414$ & $0.1697 / 0.1934$ & $0.1507 / 0.1820$ \\
\hline \multicolumn{8}{|l|}{ No. of atoms } \\
\hline Overall & 41700 & 42006 & 39500 & 36620 & 36629 & 40595 & 40439 \\
\hline Protein & 35426 & 35538 & 34751 & 34668 & 34508 & 34826 & 34712 \\
\hline Ligands & 598 & 357 & 392 & 320 & 272 & 320 & 330 \\
\hline Water & 5676 & 6111 & 4357 & 1632 & 1849 & 5449 & 5397 \\
\hline RMS (bonds) (̊̊) & 0.008 & 0.006 & 0.007 & 0.008 & 0.008 & 0.008 & 0.007 \\
\hline RMS (angles) $\left(^{\circ}\right.$ ) & 1.070 & 1.062 & 1.076 & 1.150 & 1.156 & 0.972 & 1.070 \\
\hline \multicolumn{8}{|c|}{ Ramachandran plot } \\
\hline Favored (\%) & 97.81 & 97.69 & 98.14 & 98.00 & 97.95 & 97.70 & 97.96 \\
\hline Allowed (\%) & 2.01 & 1.20 & 1.73 & 1.98 & 2.03 & 2.14 & 1.86 \\
\hline Outliers (\%) & 0.18 & 0.11 & 0.13 & 0.02 & 0.02 & 0.16 & 0.18 \\
\hline \multicolumn{8}{|c|}{ Average $B$-factor $\left(\AA^{2}\right)$} \\
\hline Overall & 17.4 & 17.1 & 19.1 & 26.0 & 17.0 & 17.7 & 19.3 \\
\hline Protein & 15.2 & 14.8 & 17.9 & 25.8 & 16.8 & 15.5 & 17.4 \\
\hline Ligands & 20.1 & 15.2 & 21.1 & 27.7 & 15.7 & 21.5 & 19.3 \\
\hline Solvent & 30.9 & 30.6 & 29.1 & 29.9 & 21.6 & 31.5 & 31.5 \\
\hline
\end{tabular}

a. $R$ merge $=\Sigma\left|\mathrm{Ii}_{\mathrm{i}}-\mathrm{Im}\right| / \Sigma \mathrm{I}$, where $\mathrm{I}_{\mathrm{i}}$ is the intensity of the measured reflection and $\mathrm{Im}$ is the mean intensity of all symmetry related reflections.

b. $R_{\text {work }}=\Sigma|| \mathrm{F}_{\text {obs }}|-| \mathrm{F}_{\text {calc }}|/ \Sigma| \mathrm{F}_{\text {obs }} \mid$, where $\mathrm{F}_{\text {obs }}$ and $\mathrm{F}_{\text {calc }}$ are observed and calculated structure factors. $R$ free $=\Sigma \mathrm{T}|| \mathrm{F}_{\text {obs }}|-| \mathrm{F}_{\text {calc }}|/ \Sigma \mathrm{T}| \mathrm{F}_{\text {obs }} \mid$, where $\mathrm{T}$ is a test data set of about $5 \%$ randomly chosen reflections excluded from the refinement. Numbers in parentheses represent the value for the highest resolution shell. 
Table S2. $C_{2 \alpha}$ stereochemistry and relevant parameters in enzyme-free or enzyme-bound tetrahedral adducts with ThDP or 3-deaza ThDP. ${ }^{a}$

\begin{tabular}{|c|c|c|c|c|c|c|c|}
\hline PDB ID ${ }^{b}$ & $\begin{array}{l}\text { Stereochemistry } \\
\left(C_{2 \alpha}\right)\end{array}$ & $\mathrm{C}_{2}-\mathrm{C}_{2 \alpha}(\AA)$ & $N_{4^{\prime}}-C_{2 \alpha}(\AA)$ & $\mathrm{O}_{2 \alpha}-\mathrm{N}_{4^{\prime}}(\AA)$ & $\mathrm{O}_{2 \alpha}-\mathrm{C}_{2 \alpha}-\mathrm{C}_{2}\left(^{\circ}\right)$ & $\mathrm{O}_{2 \alpha}-\mathrm{C}_{2 \alpha}-\mathrm{C}_{2 \beta}\left(^{\circ}\right)$ & $\mathrm{C}_{2}-\mathrm{C}_{2 \alpha}-\mathrm{C}_{2 \beta}\left({ }^{\circ}\right)$ \\
\hline 5EJ8 (MenD) & $S$ & 1.49 & 3.03 & 4.36 & 94.19 & 98.42 & 113.46 \\
\hline 3DUF & $R$ & 1.53 & 3.32 & 2.30 & 109.81 & 111.15 & 109.04 \\
\hline $2 \mathrm{VBG}$ & $R$ & 1.53 & 3.56 & 2.95 & 98.78 & 110.06 & 119.07 \\
\hline $2 \mathrm{Q} 5 \mathrm{~L}^{c}$ & $R$ & 1.54 & 3.37 & 2.60 & 110.98 & 112.43 & 110.84 \\
\hline $2 \mathrm{Q} 5 \mathrm{~L}^{c}$ & $S$ & 1.55 & 3.44 & 2.59 & 112.77 & 114.25 & 112.18 \\
\hline 3ZHS & $S$ & 1.53 & 3.76 & 2.89 & 113.26 & 108.49 & 112.34 \\
\hline LIWREO & $S$ & 1.50 & 5.20 & 6.53 & 110.30 & 102.60 & 111.78 \\
\hline 1GPU & --- & 1.27 & 2.95 & 2.96 & 132.79 & 119.46 & 107.75 \\
\hline
\end{tabular}
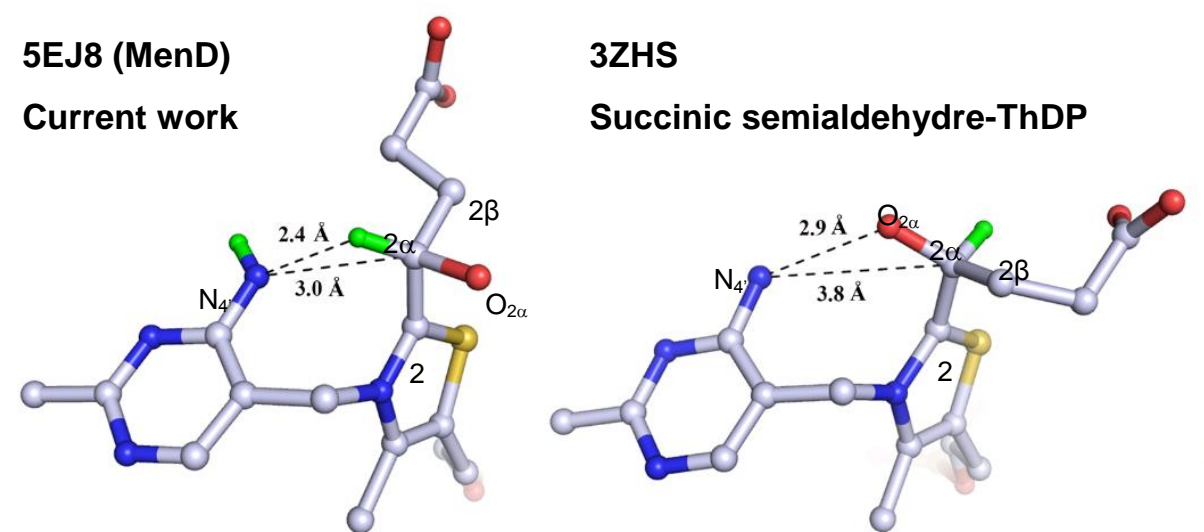

3DUF

2-(1-hydroxyethyl)-3-deaza-ThDP

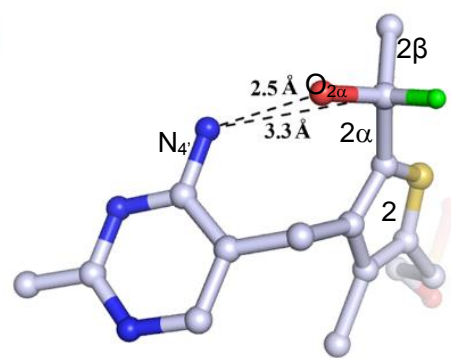

a. The adduct structures in 5EJ8 (Model II), 3ZHS and 3DUF are presented below the table with the N, $\mathrm{H}$, $\mathrm{O}, \mathrm{S}$ and $\mathrm{C}$ atoms in blue, green, red, gold and gray, respectively.

$b$. Only the ligand in one subunit (chain A) of the crystal structures is used; LIWREO is an enzyme-free ThDP adduct deposited in the Cambridge Crystallographic Data Centre (CCDC) (available at http://www.ccdc.cam.ac.uk/); An enamine intermediate from 1GPU is included for comparison.

c. Both conformations are modeled for 2-(1-hydroxyethyl)-3-deaza-ThDP in different subunits of 2Q5L. 

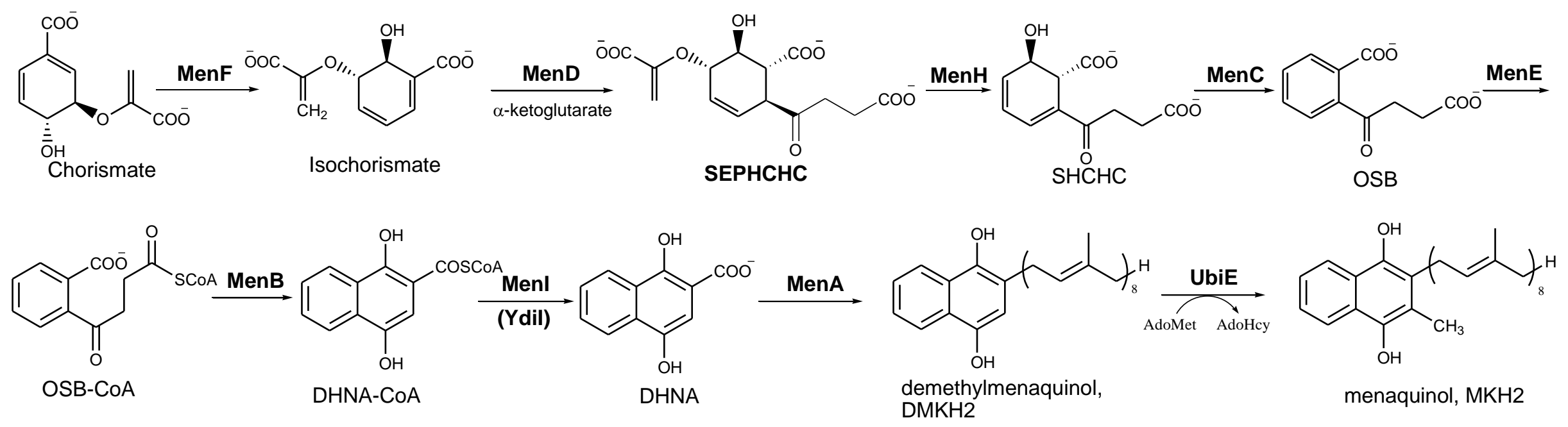

\section{Biosynthetic genes}

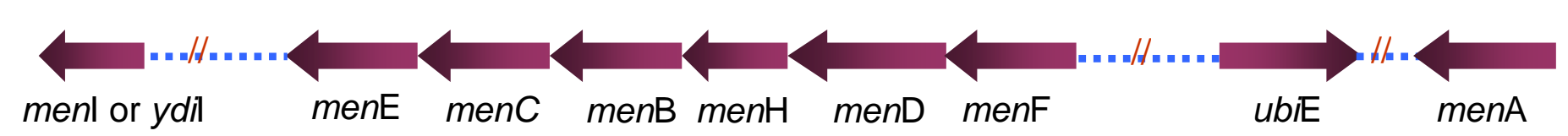

Figure S1. The biosynthesis of menaquinone (vitamin K2) in Escherichia coli. SHCHC: $(1 R, 6 R)$-2-succinyl-6-hydroxy-2, 4-cyclohexadiene-1carboxylate; SEPHCHC:(1R, 2S, 5S, 6S)-2-succinyl-5-enolpyruvyl-6- hydroxy-3-cyclohexene-1-carboxylate; OSB: $o$-succinyl-1-benzoate; DHNA: 1, 4-dihydroxy-2-naphthanoate; CoA: coenzyme A; DMKH2: demethylmenaquinol; MKH2: menaquinol; AdoMet: $S$-adenosyl methionine; AdoHcy: $S$-adenosyl homocysteine. 

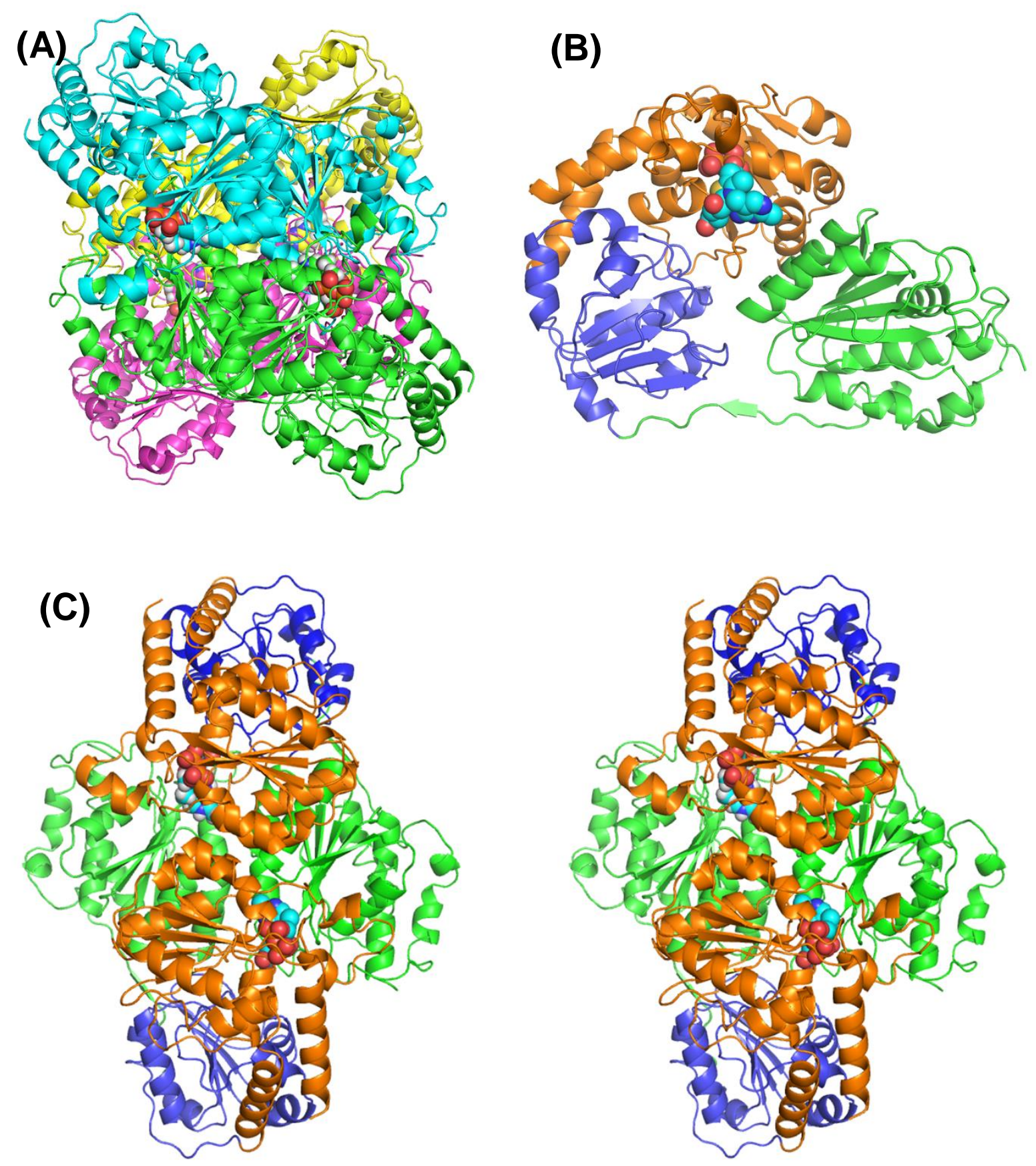

Figure S2. Crystal structure of E. coli MenD complexed with the tetrahedral intermediate. (A) The dimer-of-dimer assembly. (B) Domain structure of a MenD subunit. (C) Stereoview of an active dimer in the MenD structure. The structures are drawn from PDB ID 5EJ8 at $1.34 \AA$ resolution and are colored by chains in $(\mathbf{A})$ and by domains in $(\mathbf{B})$ and $(\mathbf{C})$. The tetrahedral intermediate is represented in spheres with carbon atoms colored in cyan. 
(A)
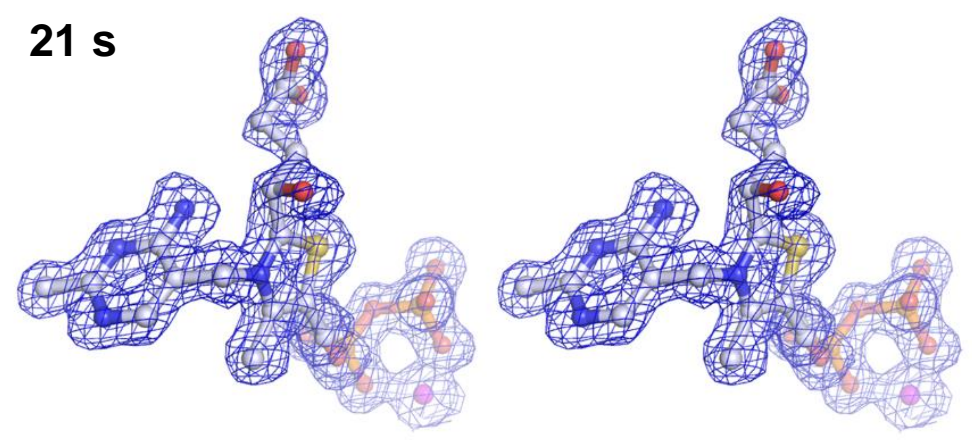

(B) 2 min
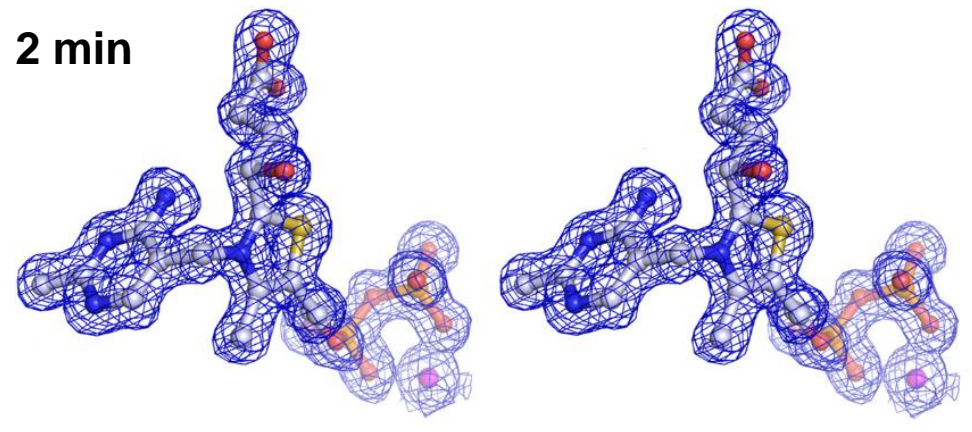

(C) $15 \mathrm{~min}$
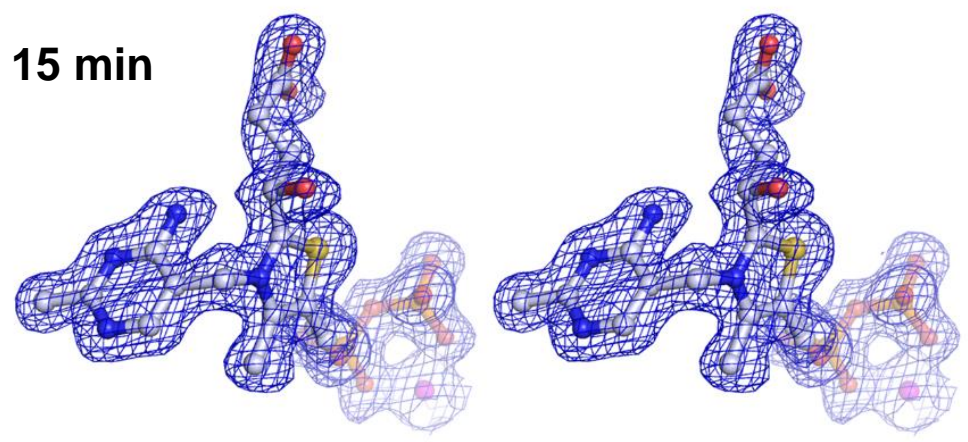

(D) $90 \mathrm{~min}$
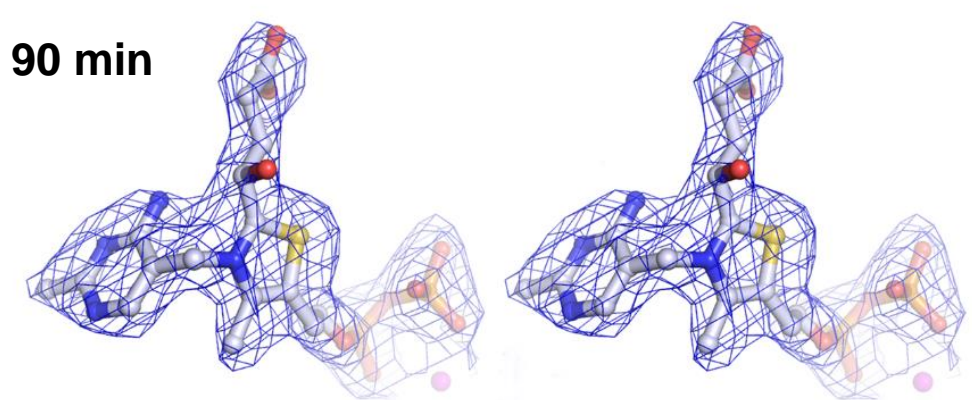

Figure S3. Stereo diagram of the $2 \mathrm{~m} F_{\mathrm{o}}-\mathrm{D} F_{\mathrm{c}}$ electron density map of the tetrahedral intermediate formed in MenD crystals after soaked with $10 \mathrm{mM} \alpha$-ketoglutarate at room temperature for a varied time period: (A) 21s (PDB entry 5EJ7); (B) 2 min (PDB entry 5EJ8); (C) 15 min (PDB entry 5EJ4); and (D) 90 min (PDB entry 5EJ5). The maps are contoured at $1.0 \sigma$ in blue mesh and $(\mathbf{B})$ is the same as in Figure $2(\mathbf{C})$ except at a different contour level. The tetrahedral intermediate is represented in sticks with grey carbon atoms and the manganese (II) ion is denoted with a magenta sphere. 


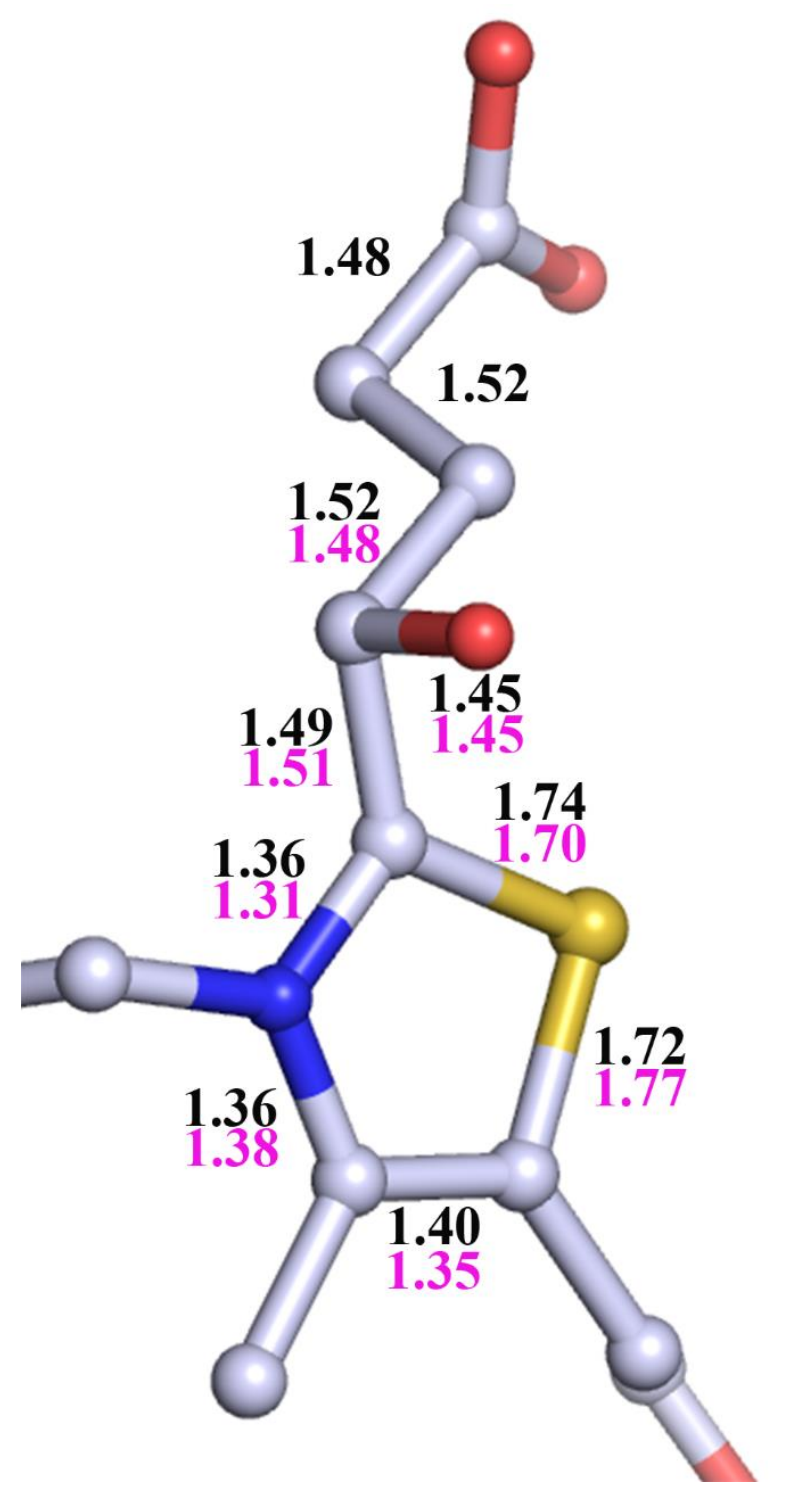

Figure S4. Lengths of chemical bonds in the thiazolium ring and the $\mathrm{C}_{2}$-appendage in the MenD-bound postdecarboxylation intermediate compared to those in an enzyme-free 2-(1hydroxyethyl)-ThDP. ${ }^{18}$ The bond lengths are indicated in black for the MenD ligand and in pink for the enzyme-free molecule. 

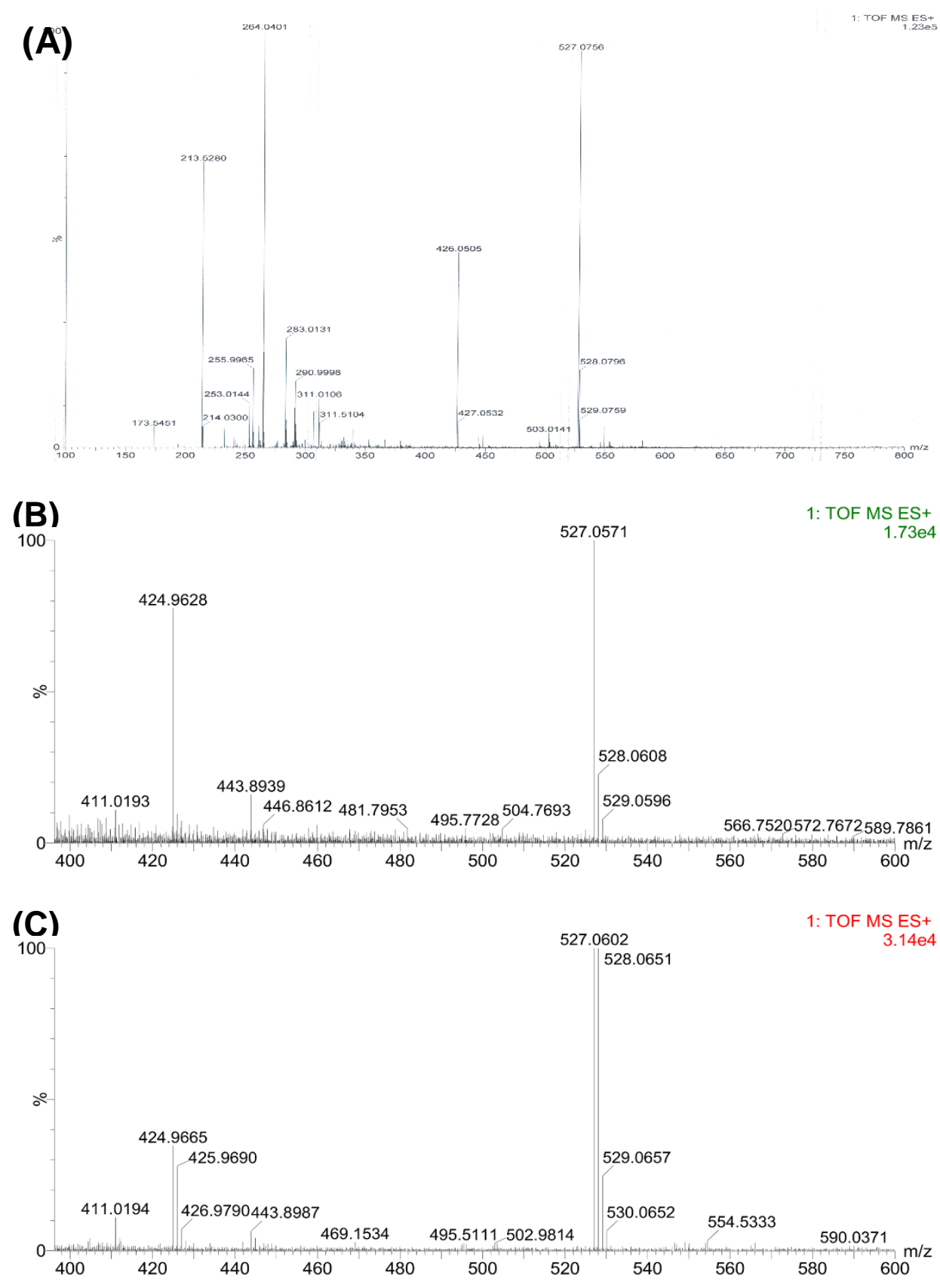

Figure S5. ESI-MS spectra of the products formed in the reaction between $\alpha$-ketoglutarate and ThDP-bound MenD. (A) Product mixture from acid quenching of a normal reaction after 1 min. (B) ThDP adduct purified from the control reaction in the deuterium exchange experiment. (C) ThDP adduct isolated from the deuterium exchange reaction in the presence of $50 \% \mathrm{D}_{2} \mathrm{O}$. Preparation and purification of the ThDP adduct in $(\mathbf{B})$ and $(\mathbf{C})$ are described in the Materials and Methods. Calculated exact molecular weights: ThDP $\left(\mathrm{C}_{12} \mathrm{H}_{19} \mathrm{~N}_{4} \mathrm{O}_{7} \mathrm{P}_{2} \mathrm{~S}^{+}\right)$, 425.04; succinic semialdehyde-ThDP adduct $\left(\mathrm{C}_{16} \mathrm{H}_{25} \mathrm{~N}_{4} \mathrm{O}_{10} \mathrm{P}_{2} \mathrm{~S}^{+}\right), \quad 527.08 ; \quad \mathrm{C} 2 \alpha$-deuterated succinic semialdehyde-ThDP adduct $\left(\mathrm{C}_{16} \mathrm{H}_{24} \mathrm{DN}_{4} \mathrm{O}_{10} \mathrm{P}_{2} \mathrm{~S}^{+}\right), 528.08$. 
(A)

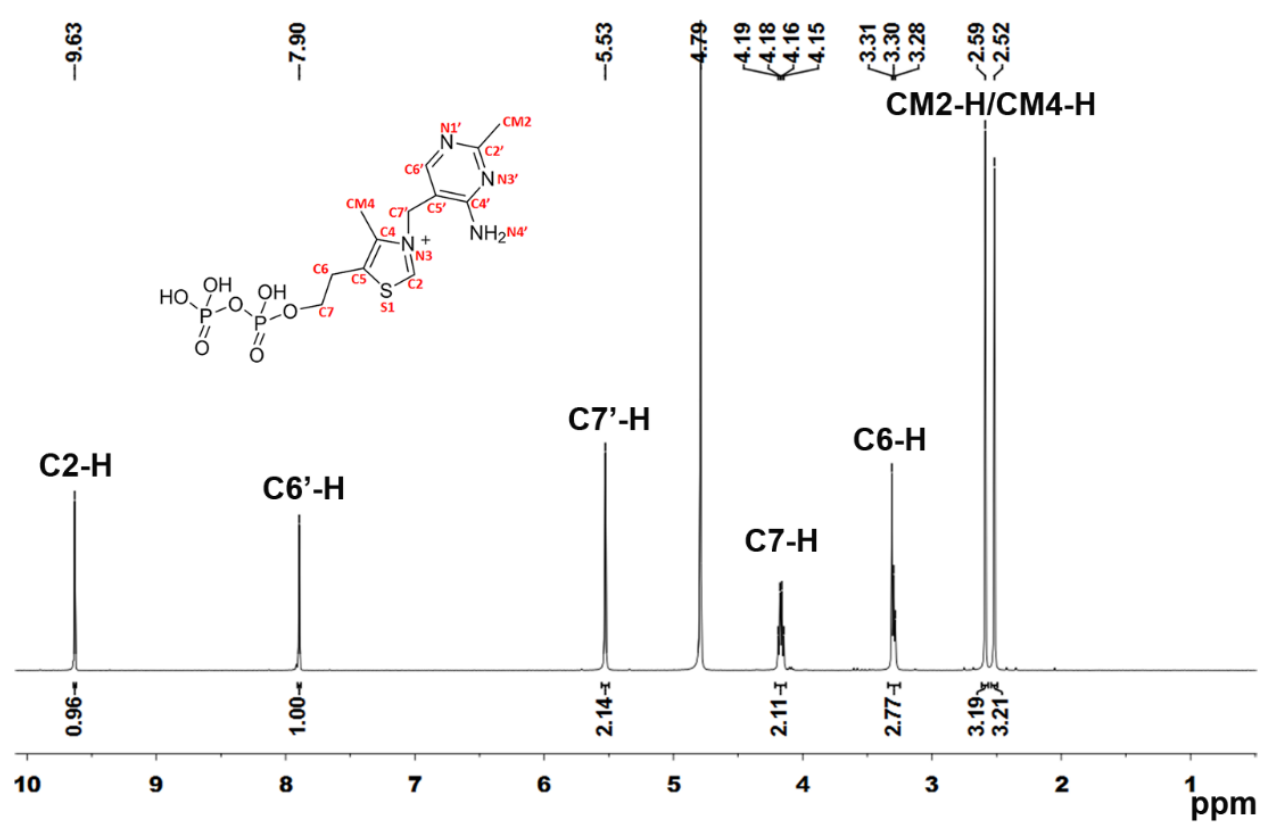

(B)

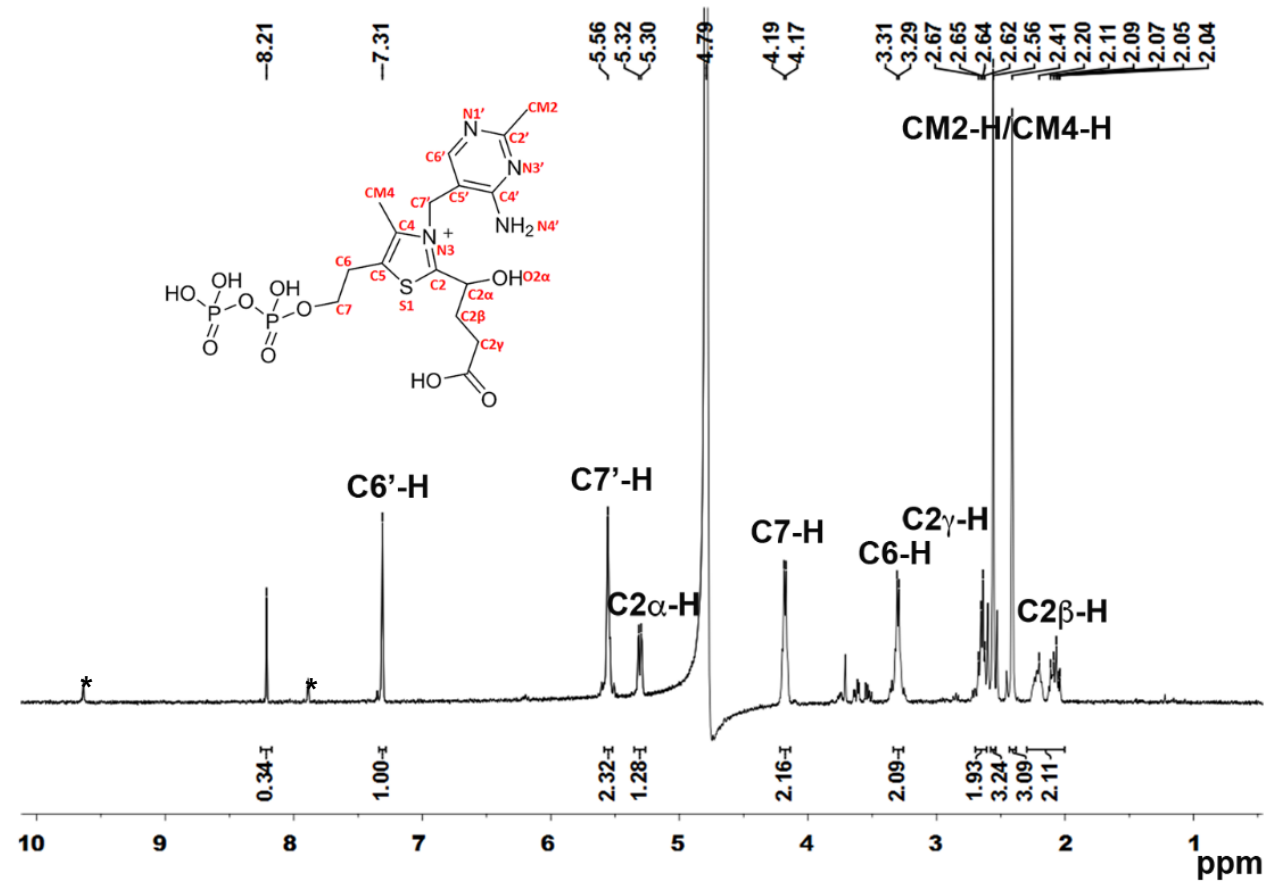

(C)

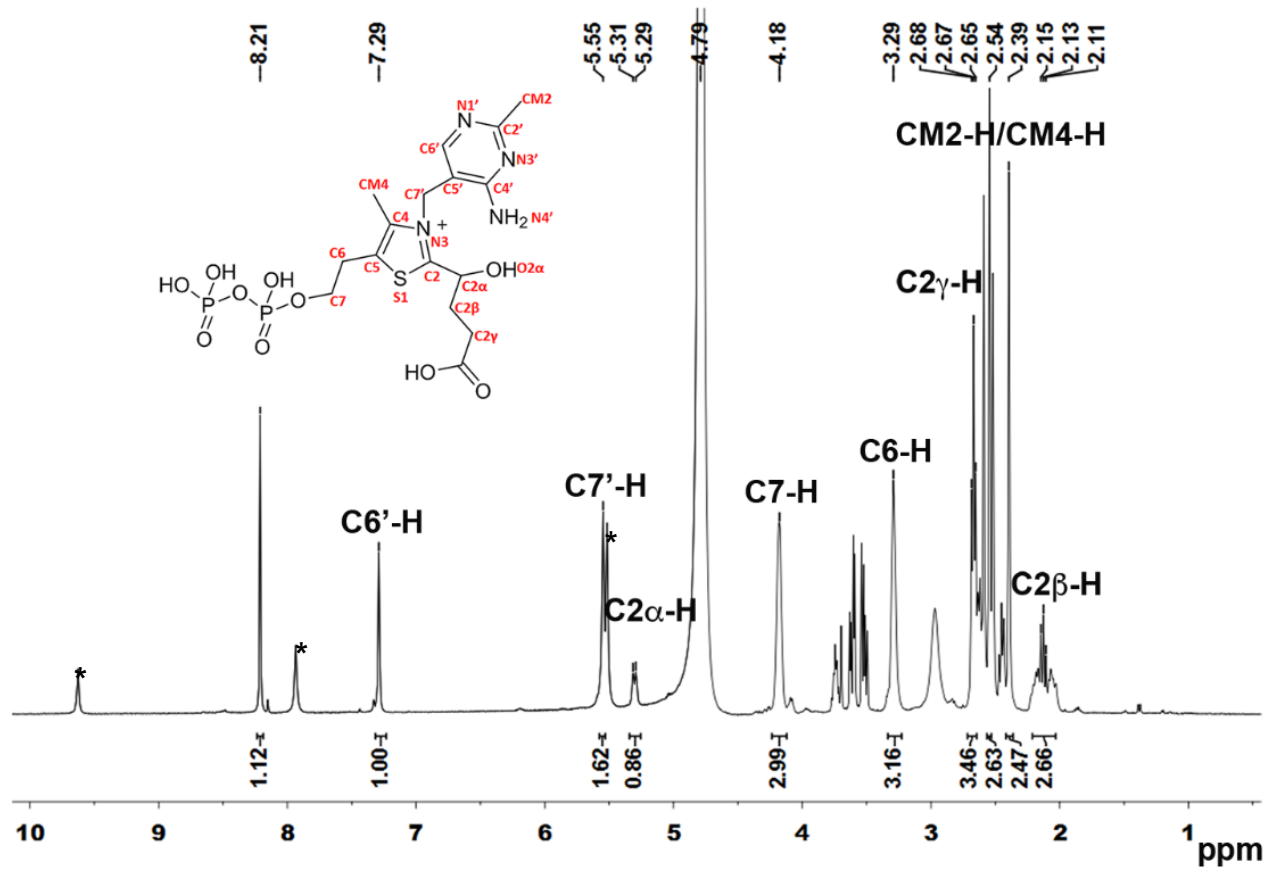

(See next page for the figure legend) 
Figure S6. ${ }^{1} \mathrm{H}-\mathrm{NMR}$ spectra of ThDP and the succinic semialdehyde-ThDP adduct from the deuterium exchange experiment. (A) ${ }^{1} \mathrm{H}-\mathrm{NMR}$ spectrum of commercially available ThDP with signals properly assigned according to Spectral Database for Organic Compounds (SDBS) [National Institute of Advanced Industrial Science and Technology (AIST). ${ }^{1} \mathrm{H}-\mathrm{NMR}$ spectrum; SDBS No.: 4986; HSP-41-999; http://sdbs.db.aist.go.jp (accessed Nov 26, 2015)]. (B) ${ }^{1} \mathrm{H}-$ NMR spectrum of the ThDP adduct purified from the control reaction in the deuterium exchange experiment. (C) ${ }^{1} \mathrm{H}-\mathrm{NMR}$ spectrum of the ThDP isolated from the deuterium exchange reaction in the presence of $50 \% \mathrm{D}_{2} \mathrm{O}$. All samples were dissolved in $\mathrm{D}_{2} \mathrm{O}$ for the spectrum recording. The ThDP adduct in $(\mathbf{B})$ and $(\mathbf{C})$ was obtained and purified by HPLC from the deuterium exchange reactions described in the Materials and Methods. The $\mathrm{C}_{6}{ }^{-}-\mathrm{H}$ signal at $\delta 7.31$ (B) or $\delta 7.29(\mathbf{C})$ was used as an internal standard to calculate the percentage of deuterium exchange at $\mathrm{C}_{2 \alpha}-\mathrm{H}$ due to its sensitivity to the identity of the covalent ThDP adduct ${ }^{19}$ Chemical shifts of $\mathrm{C}_{6}{ }^{\prime}-\mathrm{H}, \mathrm{C}_{2 \alpha}-\mathrm{H}$ and other protons were assigned by comparison to the ${ }^{1} \mathrm{H}-\mathrm{NMR}$ signals of similar ThDP adducts reported earlier. ${ }^{20-22}$ The peaks with an asterisk $(*)$ are from free ThDP while the signal at $\delta 8.21$ is from an unknown impurity and the peaks at $\delta 3.0$ and $\delta 3.50-3.75$ are from glycerol in $(\mathbf{B})$ and $(\mathbf{C})$. 


\section{Supplementary References:}

1. DeLano, W. L. The PyMOL Molecular Graphics System, DeLano Scientific, San Carlos, CA (2002).

2. Jiang, M.; Cao, Y.; Guo, Z. F.; Chen, M.; Chen, X.; Guo, Z. Biochemistry 2007, 46, 10979.

3. Liu, J.; Quinn, N.; Berchtold, G. A.; Walsh, C. T. Biochemistry 1990, 29, 1417.

4. Grisostomi, C.; Kast, P.; Pulido, R.; Huynh, J.; Hilvert, D. Bioorg. Chem. 1997, 25, 297.

5. Jiang, M.; Chen, X.; Guo, Z. F.; Cao, Y.; Chen, M.; Guo, Z. Biochemistry 2008, 47, 3426.

6. Sun, Y.; Yin, S.; Feng, Y.; Li, J.; Zhou, J.; Liu, C.; Zhu, G.; Guo, Z. J. Biol. Chem.2014, 289, 15867.

7. Otwinowski, Z.; Minor, W. Method. Enzymol.1997, 276, 307.

8. Battye, T. G. G.; Kontogiannis, L.; Johnson, O.; Powell, H. R.; Leslie, A. G. W. Acta Crystallogr. Sect. D: Biol. Crystallogr. 2011, 67, 271.

9. Evans, P. R.; Murshudov, G. N. Acta Crystallogr Sect. D: Biol. Crystallogr. 2013, 69, 1204.

10. Mccoy, A. J.; Grosse-Kunstleve, R. W.; Adams, P. D.; Winn, M. D.; Storoni, L. C.; Read, R. J. J. Appl. Crystallogr. 2007,40, 658.

11. Dawson, A.; Fyfe, P. K.; Hunter, W. N. J. Mol. Biol. 2008, 384, 1353.

12. Emsley, P.; Lohkamp, B.; Scott, W. G.; Cowtan, K. Acta Crystallogr. Sect. D: Biol. Crystallogr. 2010, 66, 486.

13. Adams, P. D.; Afonine, P. V.; Bunkoczi, G.; Chen, V. B.; Davis, I. W.; Echols, N.; Headd, J. J.; Hung, L. W.; Kapral, G. J.; Grosse-Kunstleve, R. W.; McCoy, A. J.; Moriarty, N. W.; Oeffner, R.; Read, R. J.; Richardson, D. C.; Richardson, J. S.; Terwilliger, T. C.; Zwart, P. H. Acta Crystallogr. Sect. D: Biol. Crystallogr. 2010, 66, 213.

14. Schuttelkopf, A. W.; van Aalten, D. M.; Acta Crystallogr. Sect. D: Biol. Crystallogr. 2004, 60, 1355.

15. Moriarty, N. W.; Grosse-Kunstleve, R. W.; Adams, P. D. Acta Crystallogr. Sect. D: Biol. Crystallogr. 2009, 65, 1074.

16. Laskowski, R. A.; Macarthur, M. W.; Moss, D. S.; Thornton, J. M. J. Appl. Crystallogr. 1993, 26, 283.

17. Chen, V. B.; Arendall, W. B.; Headd, J. J.; Keedy, D. A.; Immormino, R. M.; Kapral, G. J.; Murray, L. W.; Richardson, J. S.; Richardson, D. C. Acta Crystallogr. Sect. D: Biol. Crystallogr. 2010, 66, 12.

18. Malandrinos, G.; Louloudi, M.; Mitsopoulou, C. A.; Butler, I. S.; Bau, R.; Hadjiliadis, N.; J. Biol. Inorg. Chem. 1998, 3, 437.

19. Tittmann, K.; Golbik, R.; Uhlemann, K.; Khailova, L.; Schneider, G.; Patel, M.; Jordan, F.; Chipman, D. M.; Duggleby, R. G.; Hubner, G. Biochemistry 2003, 42, 7885.

20. Lautens, J. C.; Kluger, R. J. Org. Chem. 1992, 57, 6410.

21. Balakrishnan, A.; Nemeria, N. S.; Chakraborty, S.; Kakalis, L.; Jordan, F. J. Am. Chem. Soc. 2012, 134, 18644.

22. Wille, G.; Meyer, D.; Steinmetz, A.; Hinze, E.; Golbik, R.; Tittmann, K. Nat. Chem. Biol. 2006, 2, 324. 